\title{
Process-Property Relationships for Melt-Spun Poly(lactic acid) Yarn
}

Chirag R. Gajjar, ${ }^{\dagger}$ Jon W. Stallrich, ${ }^{\ddagger}$ Melissa A. Pasquinelli ${ }^{\dagger, \S}$ and Martin W. King*,†,»

†Wilson College of Textiles, North Carolina State University, Raleigh 27606, United States

${ }^{\ddagger}$ Department of Statistics, North Carolina State University, Raleigh 27695, United States

${ }^{\S}$ College of Natural Resources, North Carolina State University, Raleigh 27695, United States

"College of Textiles, Donghua University, Songjiang District, Shanghai 201620, China

*Email: mwking2@ncsu.edu. Tel: +1-919-291-2563. 


\section{Statistical Analysis for Process-Property Relationships:}

Statistical analysis was performed using JMP Pro 14 software (SAS Institute). A two-stage analysis was performed on the drawn samples. First, we averaged over the settings of the drawing parameters ( $D$ and $R$ ) and the important melt extrusion parameters were determined using halfnormal plot. After this analysis, a split-plot analysis was performed including main effects and two-factor interaction effects between the important melt extrusion parameters and the two drawing parameters, the latter being the split-plot factors. The split-plot analysis also entertained the possibility of three-factor interaction effects between the melt extrusion parameters and drawing parameters. The split-plot effects had higher power, so effects having $p$-values less than $\alpha=0.05$ were deemed important, while for whole-plot effects we used $\alpha=0.10$ (since the tests were prone to low power due to the limited number of samples). For the max draw ratio analysis, an $\alpha=0.10$ cutoff was used for the split-plot effects since there were only 16 samples, not 32 . Effects found to be statistically insignificant were then removed from the model unless they violated heredity rules. For example, if the three-factor interaction PDR were significant then we included all three main effects PDR and all three two-factor interactions PD, PR, and DR, even if they are statistically insignificant.

Table S1: Raw data for Fixed Effect Tests and Final Regression Model for response variables in the study 


\begin{tabular}{|c|c|c|c|c|c|c|}
\hline $\begin{array}{l}\text { Response } \\
\text { Variable }\end{array}$ & Fixed Eff & $\begin{array}{l}\text { Tests from } \\
\text { If-normal }\end{array}$ & $\begin{array}{l}\text { itial split-I } \\
\text { ot analysis }\end{array}$ & $\begin{array}{l}\text { model usi } \\
\text { d the Fina }\end{array}$ & $\begin{array}{l}\text { significant fa } \\
\text { Regression Mc }\end{array}$ & $\begin{array}{l}\text { ors from the } \\
\text { el }\end{array}$ \\
\hline \multirow{10}{*}{$\begin{array}{l}\text { Maximum } \\
\text { Draw Ratio }\end{array}$} & \multicolumn{4}{|c|}{ Fixed Effect Tests } & \multicolumn{2}{|c|}{ Final Regression Model } \\
\hline & Effect & $\begin{array}{c}\text { Denom } \\
\text { DF }\end{array}$ & F Ratio & $p$-value & $\begin{array}{c}\text { Parameter } \\
\text { Estimate }\end{array}$ & $\begin{array}{l}\text { Standard } \\
\text { Error }\end{array}$ \\
\hline & Intercept & -- & -- & -- & 2.37 & 0.086 \\
\hline & $\mathrm{T}$ & 4 & 10.69 & 0.0309 & -0.26 & 0.086 \\
\hline & $\mathrm{P}$ & 4 & 36.84 & 0.0037 & 0.49 & 0.086 \\
\hline & $\mathrm{T}^{*} \mathrm{P}$ & 4 & 1.66 & 0.2663 & 0.60 & 0.244 \\
\hline & $\mathrm{D}$ & 4 & 7.34 & 0.0534 & -- & -- \\
\hline & $\mathrm{D} * \mathrm{~T}$ & 4 & 2.22 & 0.2102 & -- & -- \\
\hline & $\mathrm{D} * \mathrm{P}$ & 4 & 0.14 & 0.7331 & -- & -- \\
\hline & $\mathrm{T} * \mathrm{P} * \mathrm{D}$ & 4 & 2.10 & 0.2202 & -- & -- \\
\hline
\end{tabular}




\begin{tabular}{|c|c|c|c|c|c|c|}
\hline $\begin{array}{c}\text { Response } \\
\text { Variable }\end{array}$ & \multicolumn{6}{|c|}{$\begin{array}{l}\text { Fixed Effect Tests from initial split-plot model using significant factors from the } \\
\text { half-normal plot analysis and the Final Regression Model }\end{array}$} \\
\hline \multirow{17}{*}{ Yarn Size } & \multicolumn{4}{|c|}{ Fixed Effect Tests } & \multicolumn{2}{|c|}{ Final Regression Model } \\
\hline & Effect & $\begin{array}{c}\text { Denom } \\
\text { DF }\end{array}$ & F Ratio & $p$-value & $\begin{array}{c}\text { Parameter } \\
\text { Estimate }\end{array}$ & $\begin{array}{l}\text { Standard } \\
\text { Error }\end{array}$ \\
\hline & Intercept & -- & -- & -- & 33.15 & 0.257 \\
\hline & $\mathrm{P}$ & 4 & 1841.66 & $<0.0001$ & 11.02 & 0.257 \\
\hline & $\mathrm{S}$ & 4 & 3900.24 & $<0.0001$ & -16.04 & 0.257 \\
\hline & $\mathrm{P} * \mathrm{~S}$ & 4 & 268.09 & $<0.0001$ & -4.20 & 0.257 \\
\hline & $\mathrm{D}$ & 13 & 1979.53 & $<0.0001$ & -14.57 & 0.325 \\
\hline & $\mathrm{R}$ & 13 & 45.82 & $<0.0001$ & -2.37 & 0.341 \\
\hline & $\mathrm{P}^{*} \mathrm{D}$ & 13 & 353.70 & $<0.0001$ & -6.16 & 0.325 \\
\hline & $\mathrm{P} * \mathrm{R}$ & 13 & 0.36 & 0.5576 & 0.24 & 0.341 \\
\hline & S*D & 13 & 854.03 & $<0.0001$ & 9.57 & 0.325 \\
\hline & $\mathrm{S} * \mathrm{R}$ & 13 & 2.08 & 0.1733 & 0.53 & 0.341 \\
\hline & $\mathrm{D} * \mathrm{R}$ & 13 & 8.17 & 0.0135 & -0.96 & 0.341 \\
\hline & $P * S * D$ & 13 & 101.90 & $<0.0001$ & 3.31 & 0.325 \\
\hline & $\mathrm{P} * \mathrm{~S} * \mathrm{R}$ & 13 & 0.77 & 0.3966 & - & -- \\
\hline & $\mathrm{P} * \mathrm{D} * \mathrm{R}$ & 13 & 5.29 & 0.0387 & -0.82 & 0.341 \\
\hline & $\mathrm{S} * \mathrm{D} * \mathrm{R}$ & 13 & 11.20 & 0.0053 & 1.13 & 0.341 \\
\hline
\end{tabular}




\begin{tabular}{|c|c|c|c|c|c|c|}
\hline $\begin{array}{l}\text { Response } \\
\text { Variable }\end{array}$ & Fixed Effe & $\begin{array}{l}\text { ests from } \\
\text { If-normal }\end{array}$ & $\begin{array}{l}\text { itial split- } \\
\text { ot analysis }\end{array}$ & model usi & $\begin{array}{l}\text { significant } f \\
\text { egression } M\end{array}$ & ors from the \\
\hline \multirow{17}{*}{ Crystallinity } & \multicolumn{4}{|c|}{ Fixed Effect Tests } & \multicolumn{2}{|c|}{ Final Regression Model } \\
\hline & Effect & $\begin{array}{c}\text { Denom } \\
\text { DF }\end{array}$ & F Ratio & $p$-value & $\begin{array}{l}\text { Parameter } \\
\text { Estimate }\end{array}$ & $\begin{array}{c}\text { Standard } \\
\text { Error }\end{array}$ \\
\hline & Intercept & -- & -- & -- & 38.26 & 0.486 \\
\hline & $\mathrm{P}$ & 4 & 9.86 & 0.0347 & -1.53 & 0.486 \\
\hline & $S$ & 4 & 89.49 & 0.0007 & 4.60 & 0.486 \\
\hline & $\mathrm{P} * \mathrm{~S}$ & 4 & 3.46 & 0.1356 & -0.91 & 0.486 \\
\hline & $\mathrm{D}$ & 13 & 616.53 & $<0.0001$ & 13.39 & 0.524 \\
\hline & $\mathrm{R}$ & 13 & 29.27 & 0.0001 & 3.05 & 0.550 \\
\hline & $\mathrm{P} * \mathrm{D}$ & 13 & 7.78 & 0.0152 & 1.51 & 0.524 \\
\hline & $\mathrm{P} * \mathrm{R}$ & 13 & 0.88 & 0.3647 & -0.51 & 0.550 \\
\hline & S*D & 13 & 0.26 & 0.6155 & 0.28 & 0.524 \\
\hline & $\mathrm{S} * \mathrm{R}$ & 13 & 6.35 & 0.0258 & -1.40 & 0.550 \\
\hline & $\overline{D * R}$ & 13 & 15.29 & 0.0018 & 2.25 & 0.550 \\
\hline & $\mathrm{P} * \mathrm{~S} * \mathrm{D}$ & 13 & 4.88 & 0.0452 & -1.19 & 0.524 \\
\hline & $\mathrm{P} * \mathrm{~S} * \mathrm{R}$ & 13 & 0.22 & 0.6432 & -- & -- \\
\hline & $\mathrm{P} * \mathrm{D} * \mathrm{R}$ & 13 & 4.75 & 0.0481 & 1.21 & 0.550 \\
\hline & S*D*R & 13 & 17.39 & 0.0011 & -2.40 & 0.550 \\
\hline
\end{tabular}




\begin{tabular}{|c|c|c|c|c|c|c|}
\hline $\begin{array}{l}\text { Response } \\
\text { Variable }\end{array}$ & \multicolumn{6}{|c|}{$\begin{array}{l}\text { Fixed Effect Tests from initial split-plot model using significant factors from the } \\
\text { half-normal plot analysis and the Final Regression Model }\end{array}$} \\
\hline \multirow{17}{*}{$\mathrm{M}_{\mathrm{w}}$} & \multicolumn{4}{|c|}{ Fixed Effect Tests } & \multicolumn{2}{|c|}{ Final Regression Model } \\
\hline & Effect & $\begin{array}{c}\text { Denom } \\
\text { DF }\end{array}$ & F Ratio & $p$-value & $\begin{array}{l}\text { Parameter } \\
\text { Estimate }\end{array}$ & $\begin{array}{l}\text { Standard } \\
\text { Error }\end{array}$ \\
\hline & Intercept & -- & -- & -- & -0.16 & 0.002 \\
\hline & $\mathrm{T}$ & 4 & 18.13 & 0.0131 & -0.01 & 0.002 \\
\hline & $\mathrm{S}$ & 4 & 38.88 & 0.0034 & -0.01 & 0.002 \\
\hline & $\mathrm{T} * \mathrm{~S}$ & 4 & 2.74 & 0.1733 & -- & -- \\
\hline & $\mathrm{D}$ & 13 & 20.16 & 0.0006 & -0.02 & 0.003 \\
\hline & $\mathrm{R}$ & 13 & 0.03 & 0.8669 & -- & -- \\
\hline & T*D & 13 & 4.70 & 0.0493 & 0.01 & 0.003 \\
\hline & $\mathrm{T}^{*} \mathrm{R}$ & 13 & 2.25 & 0.1576 & -- & -- \\
\hline & S*D & 13 & 0.34 & 0.5680 & -- & -- \\
\hline & $\mathrm{S} * \mathrm{R}$ & 13 & 0.78 & 0.3927 & -- & -- \\
\hline & $\mathrm{D} * \mathrm{R}$ & 13 & 0.34 & 0.5695 & -- & -- \\
\hline & $\mathrm{T} * \mathrm{~S} * \mathrm{D}$ & 13 & 2.54 & 0.1351 & -- & -- \\
\hline & $\mathrm{T} * \mathrm{~S} * \mathrm{R}$ & 13 & 0.00 & 0.9956 & - & -- \\
\hline & $\mathrm{T} * \mathrm{D} * \mathrm{R}$ & 13 & 0.97 & 0.3422 & -- & -- \\
\hline & $\mathrm{S} * \mathrm{D} * \mathrm{R}$ & 13 & 0.15 & 0.7056 & -- & -- \\
\hline
\end{tabular}




\begin{tabular}{|c|c|c|c|c|c|c|}
\hline $\begin{array}{l}\text { Response } \\
\text { Variable }\end{array}$ & \multicolumn{6}{|c|}{$\begin{array}{l}\text { Fixed Effect Tests from initial split-plot model using significant factors from the } \\
\text { half-normal plot analysis and the Final Regression Model }\end{array}$} \\
\hline \multirow{24}{*}{ Tenacity } & \multicolumn{4}{|c|}{ Fixed Effect Tests } & \multicolumn{2}{|c|}{$\begin{array}{l}\text { Final Regression Model } \\
\end{array}$} \\
\hline & Effect & $\begin{array}{l}\text { Denom } \\
\text { DF }\end{array}$ & F Ratio & $p$-value & $\begin{array}{l}\text { Parameter } \\
\text { Estimate }\end{array}$ & $\begin{array}{l}\text { Standard } \\
\text { Error }\end{array}$ \\
\hline & Intercept & -- & -- & -- & 16.31 & 0.342 \\
\hline & $\mathrm{T}$ & 2 & 33.22 & 0.0288 & -1.97 & 0.342 \\
\hline & $\mathrm{P}$ & 2 & 8.74 & 0.0979 & -1.01 & 0.342 \\
\hline & $\mathrm{S}$ & 2 & 72.64 & 0.0135 & 2.91 & 0.342 \\
\hline & T*S & 2 & 20.31 & 0.0459 & -1.54 & 0.342 \\
\hline & $\mathrm{P} * \mathrm{~S}$ & 2 & 16.77 & 0.0548 & -1.40 & 0.342 \\
\hline & $\mathrm{D}$ & 8 & 71.44 & $<0.0001$ & 8.00 & 0.850 \\
\hline & $\mathrm{R}$ & 8 & 8.27 & 0.0206 & 3.01 & 0.878 \\
\hline & $\mathrm{T}^{*} \mathrm{D}$ & 8 & 0.11 & 0.7462 & -- & -- \\
\hline & $\mathrm{P} * \mathrm{D}$ & 8 & 0.40 & 0.5425 & -- & -- \\
\hline & S*D & 8 & 0.75 & 0.4115 & -- & -- \\
\hline & D*R & 8 & 1.11 & 0.3228 & -- & -- \\
\hline & $\mathrm{T} * \mathrm{R}$ & 8 & 0.10 & 0.7650 & -- & -- \\
\hline & $\mathrm{P} * \mathrm{R}$ & 8 & 0.01 & 0.9377 & -- & -- \\
\hline & S*R & 8 & 0.02 & 0.8833 & -- & \begin{tabular}{l|l}
-- \\
\end{tabular} \\
\hline & $\mathrm{T} * \mathrm{~S} * \mathrm{D}$ & 8 & 0.05 & 0.8352 & -- & -- \\
\hline & $\mathrm{P} * \mathrm{~S} * \mathrm{D}$ & 8 & 1.40 & 0.2704 & -- & -- \\
\hline & $\mathrm{T} * \mathrm{~S} * \mathrm{R}$ & 8 & 0.26 & 0.6264 & -- & -- \\
\hline & $\mathrm{P} * \mathrm{~S} * \mathrm{R}$ & 8 & 0.01 & 0.9349 & -- & -- \\
\hline & $\mathrm{D} * \mathrm{R} * \mathrm{~T}$ & 8 & 0.29 & 0.6021 & -- & -- \\
\hline & $\mathrm{D} * \mathrm{R} * \mathrm{P}$ & 8 & 2.52 & 0.1512 & -- & -- \\
\hline & $\mathrm{D} * \mathrm{R} * \mathrm{~S}$ & 8 & 1.76 & 0.2213 & -- & -- \\
\hline
\end{tabular}




\begin{tabular}{|c|c|c|c|c|c|c|}
\hline $\begin{array}{l}\text { Response } \\
\text { Variable }\end{array}$ & \multicolumn{6}{|c|}{$\begin{array}{l}\text { Fixed Effect Tests from initial split-plot model using significant factors from the } \\
\text { half-normal plot analysis and the Final Regression Model }\end{array}$} \\
\hline \multirow{17}{*}{$\begin{array}{l}\text { Elongation } \\
\text { at break }\end{array}$} & \multicolumn{4}{|c|}{ Fixed Effect Tests } & \multicolumn{2}{|c|}{ Final Regression Model } \\
\hline & Effect & $\begin{array}{c}\text { Denom } \\
\text { DF }\end{array}$ & F Ratio & $p$-value & $\begin{array}{c}\text { Parameter } \\
\text { Estimate }\end{array}$ & $\begin{array}{l}\text { Standard } \\
\text { Error }\end{array}$ \\
\hline & Intercept & -- & -- & -- & 108.85 & 8.841 \\
\hline & $\mathrm{P}$ & 4 & 19.66 & 0.0114 & -39.20 & 8.841 \\
\hline & $\mathrm{S}$ & 4 & 18.32 & 0.0128 & -37.84 & 8.841 \\
\hline & $\mathrm{P} * \mathrm{~S}$ & 4 & 55.44 & 0.0017 & 65.83 & 8.841 \\
\hline & $\mathrm{D}$ & 13 & 90.51 & $<0.0001$ & -46.41 & 5.217 \\
\hline & $\mathrm{R}$ & 13 & 7.19 & 0.0189 & -14.35 & 5.388 \\
\hline & $\mathrm{P} * \mathrm{D}$ & 13 & 57.79 & $<0.0001$ & 37.08 & 5.217 \\
\hline & $\mathrm{P} * \mathrm{R}$ & 13 & 0.10 & 0.7607 & -- & -- \\
\hline & $S * D$ & 13 & 8.75 & 0.0111 & 14.43 & 5.217 \\
\hline & $\mathrm{S} * \mathrm{R}$ & 13 & 1.12 & 0.3096 & -- & -- \\
\hline & $\mathrm{D} * \mathrm{R}$ & 13 & 3.55 & 0.0823 & -- & -- \\
\hline & $\mathrm{P} * \mathrm{~S} * \mathrm{D}$ & 13 & 139.78 & $<0.0001$ & -57.67 & 5.217 \\
\hline & $\mathrm{P} * \mathrm{~S} * \mathrm{R}$ & 13 & 0.58 & 0.4604 & -- & -- \\
\hline & $\mathrm{P} * \mathrm{D} * \mathrm{R}$ & 13 & 0.07 & 0.8015 & -- & -- \\
\hline & $\mathrm{S} * \mathrm{D} * \mathrm{R}$ & 13 & 2.63 & 0.1286 & -- & -- \\
\hline
\end{tabular}

